\title{
Improved fixed bed models for correlating asymmetric adsorption breakthrough curves
}

\author{
Ronbanchob Apiratikul a , Khim Hoong Chu b,* \\ ${ }^{a}$ Department of Environmental Science, Suan Sunandha Rajabhat University, Bangkok, Thailand \\ ${ }^{\mathrm{b}}$ Honeychem Research, Auckland 1142, New Zealand \\ ${ }^{*}$ Corresponding author. \\ E-mail addresses: Ronbanchob.Ap@ ssru.ac.th (R. Apiratikul); khimchu@gmail.com (K.H. Chu)
}

\begin{abstract}
Breakthrough curves of water contaminants are usually analyzed using simple fixed bed models such as the Bohart-Adams, Thomas, and Yoon-Nelson equations, which are by design symmetric. Because breakthrough data often follow an asymmetric pattern, the use of models that do not account for asymmetry could lead to poor fits, consequently resulting in erroneous estimates of breakthrough and exhaustion times. To address this issue, the Bohart-Adams, Thomas, and Yoon-Nelson models were modified by a logarithmic transformation to enhance their data fitting ability. The three modified models were found capable of providing robust fits to seven separate sets of previously reported asymmetric breakthrough data of water contaminants (fluoride, methylene blue, salicylic acid, lead, mercury, nickel, and arsenic), with reported residual root mean square error (RRMSE) values ranging from 0.019 to 0.046 . In consequence, the new models were found capable of providing reliable estimates of breakthrough and exhaustion times corresponding to any predetermined concentration level. By contrast, the three original models were found to perform poorly, reporting inferior RRMSE values ranging from 0.038 to 0.086 for data fits and providing grossly inaccurate estimates of breakthrough and exhaustion times. The new models contain only parameters that appear in the original models, and are highly flexible, being able to assume virtually all monotonically increasing sigmoid shapes. They represent a far more accurate alternative to the original models.
\end{abstract}

Keywords: asymmetric breakthrough curve; fixed bed; Bohart-Adams; Thomas; Yoon-Nelson

\section{Introduction}

Adsorption is considered a highly efficient process technology for drinking water and wastewater purification. Because practical applications of adsorption are most often realized in fixed bed mode, considerable attention has been devoted to measuring contaminant breakthrough data using laboratory-scale adsorption columns. Simple fixed bed models such as the Bohart-Adams [1], Thomas [2], and Yoon-Nelson [3] equations are frequently employed for breakthrough curve analysis. Fixed bed models based on Fick's law of diffusion for the solid phase are more realistic [4,5], but they generally require the use of specialized software and programming code. For descriptions of some of the commonly used fixed bed models, the reader is referred to a handful of review articles that have been published in the literature of water contaminant adsorption over the last 30 years [6-9]. 
The Bohart-Adams, Thomas, and Yoon-Nelson models are often used in a "correlative" modeling approach, where the model equations are fitted to breakthrough data for parameter estimation. For design purposes, it is necessary to establish empirical relationships between model parameters and system and operational conditions such as bed length, flow rate, and initial contaminant concentration. Such a correlative design approach is best exemplified by the bed-depth-service-time or BDST method, which is based on the Bohart-Adams model. The well-known book by Cooney [10] provides practical guidance on the use of the Bohart-Adams, Thomas, and BDST models for sizing activated carbon columns. The recent introductory book by Tien [11] affords a comprehensive overview of these models, which have a long history of use in breakthrough curve modeling.

A prolific research literature on the use of the Bohart-Adams, Thomas, and Yoon-Nelson models in breakthrough curve analysis exists. As examples one may cite two very recent studies [12,13] published in this Journal. Their ubiquitous applicability is attributable to several reasons. One reason is that the availability of linearized forms of the three models allows straightforward data fitting by linear regression. Another reason is that application of the Bohart-Adams and Thomas models needs little input information, for instance, they do not require knowledge of bed void fraction and adsorbent density. At the far end of the scale, not even flow rate or bed length is required by the Yoon-Nelson model, which is devoid of typical system and operational variables.

In recent years, the three popular models have encountered criticisms on two fronts. First, they have been shown to be mathematically equivalent to one another [14-16]. As such, they should not be treated separately (as they commonly are). Second, they cannot describe asymmetric breakthrough data to a significant degree of precision because their functional form is intrinsically symmetric. It is the second issue that is the subject of this article.

It is of note that the bulk of reported experimental breakthrough curves are more or less asymmetric, which is largely a result of fronting or tailing. Fronting, also known as leakage, refers to early appearance of contaminant in the effluent while tailing describes a phenomenon where the effluent exhibits a slow approach toward the influent concentration near column saturation. Indeed, it is rare to observe perfectly symmetric breakthrough curves. The inability of the three models to track asymmetric breakthrough data could lead to inaccurate estimates of breakthrough and exhaustion times. Breakthrough times are fundamental to the evaluation of adsorption column performance while exhaustion times are needed to gauge the performance of series-column systems where certain adsorbers are operated to complete saturation prior to regeneration or replacement.

There have been several previous efforts to boost the data fitting ability of the three models. One of the earlier attempts was made by Clark and colleagues [17-19], who developed a modified logistic equation that incorporates the exponent of the Freundlich isotherm, based on the fact that the Bohart-Adams model is analogous to the logistic equation. Yan et al. [20] incorporated some parameters of the Thomas model in a dose response model containing an empirical exponent. Blagojev et al. [21] enhanced the fitting ability of the Yan model by extending it to a five-parameter equation. Hu et al. [22] 
converted the Bohart-Adams, Thomas, and Yoon-Nelson models to power law forms by introducing empirical exponents to the original models.

So far, most of the model improvement efforts have adopted the same strategy: addition of one or more empirical shape parameters. This approach works well because extra parameters tend to enhance the data fitting ability of a model. The present paper presents a new way to modify the BohartAdams, Thomas, and Yoon-Nelson equations. The modification method is a new departure from the work of earlier authors as it does not rely on extra shape parameters. The three modified models contain only parameters appearing in the original models and can be linearized. Previously reported breakthrough data of water contaminants exhibiting varying degrees of curve asymmetry are used to test the new models. Specific cases examined are the fixed bed adsorption of fluoride, methylene blue, salicylic acid, lead, mercury, nickel, and arsenic by adsorbents of different types.

\section{Model development}

\subsection{The Bohart-Adams model}

This model [1], originally developed for gas phase adsorption, was reparameterized and presented by Eckenfelder [23] and Eckenfelder and Ford [24] as a design tool for sizing liquid phase activated carbon columns based on pilot test breakthrough data. A simplified version of the BohartAdams model is written as

$$
\frac{C}{C_{\mathrm{o}}}=\frac{1}{1+\exp \left(\frac{k_{\mathrm{BA}} N_{\mathrm{o}} L}{u}-k_{\mathrm{BA}} C_{\mathrm{o}} t\right)}
$$

where $C \equiv$ the effluent concentration of contaminant at time $t ; C_{\mathrm{o}} \equiv$ the feed concentration; $k_{\mathrm{BA}} \equiv$ the Bohart-Adams rate coefficient; $N_{\mathrm{o}} \equiv$ the amount of contaminant adsorbed per unit volume of packed bed; $L \equiv$ the packed bed length; and $u \equiv$ the superficial velocity. A linear form of Eq. (1) commonly used in data fitting is given by

$\ln \left(\frac{C_{\mathrm{o}}}{C}-1\right)=\frac{k_{\mathrm{BA}} N_{\mathrm{o}} L}{u}-k_{\mathrm{BA}} C_{\mathrm{o}} t$

\subsection{The Thomas model}

This model was originally developed to describe liquid phase ion-exchange in fixed bed columns [2]. A simplified form of the original Thomas model [25], which was reparameterized and presented by Reynolds [26] as a model for sizing fixed bed adsorbers, reads

$$
\frac{C}{C_{\mathrm{o}}}=\frac{1}{1+\exp \left(\frac{k_{\mathrm{T}} q_{\mathrm{o}} M}{Q}-k_{\mathrm{T}} C_{\mathrm{o}} t\right)}
$$

where $k_{\mathrm{T}} \equiv$ the Thomas rate coefficient; $q_{\mathrm{o}} \equiv$ the amount of contaminant adsorbed per unit mass of adsorbent; $M \equiv$ the adsorbent mass; and $Q \equiv$ the volumetric flow rate. Like the Bohart-Adams model, rearranging the Thomas model leads to a linear form: 
$\ln \left(\frac{C_{\mathrm{o}}}{C}-1\right)=\frac{k_{\mathrm{T}} q_{\mathrm{o}} M}{Q}-k_{\mathrm{T}} C_{\mathrm{o}} t$

\subsection{The Yoon-Nelson model}

This model, formulated for describing the breakthrough characteristics of adsorption cartridges for respiratory protection [3], may be expressed as

$\frac{C}{C_{\mathrm{o}}}=\frac{1}{1+\exp \left(k_{\mathrm{YN}} \tau-k_{\mathrm{YN}} t\right)}$

where $k_{\mathrm{YN}} \equiv$ the Yoon-Nelson rate coefficient and $\tau \equiv$ the time required for $50 \%$ breakthrough. Eq. (5) can be rearranged to give the following linear form:

$\ln \left(\frac{C_{\mathrm{o}}}{C}-1\right)=k_{\mathrm{YN}} \tau-k_{\mathrm{YN}} t$

\subsection{Mathematical equivalence}

As mentioned previously, the Bohart-Adams, Thomas, and Yoon-Nelson models are mathematically analogous to one another [14-16]. A simple way to demonstrate this mathematical equivalence is to express each model in terms of the logistic equation with two general parameters:

$\frac{C}{C_{\mathrm{o}}}=\frac{1}{1+\exp (a-b t)}$

or

$\ln \left(\frac{C_{\mathrm{o}}}{C}-1\right)=a-b t$

where the parameters $a$ and $b$ are given by

Bohart-Adams: $a=\frac{k_{\mathrm{BA}} N_{\mathrm{o}} L}{u} ; b=k_{\mathrm{BA}} C_{\mathrm{o}}$

Thomas: $a=\frac{k_{\mathrm{T}} q_{\mathrm{o}} M}{Q} ; b=k_{\mathrm{T}} C_{\mathrm{o}}$

Yoon-Nelson: $a=k_{\mathrm{YN}} \tau ; b=k_{\mathrm{YN}}$

Fitting the logistic equation given by Eq. (7) to breakthrough data will yield $a$ and $b$ from which all the unknown parameters of the three models may be computed using Eqs. (9)-(11). Accordingly, it is not necessary to fit the three models one by one to the same set of breakthrough data in order to estimate their parameters.

\subsection{Modified models}

To enhance the fitting ability of the three models, we propose that certain groups of parameters and variables in the three models be replaced with logarithmic terms. Since one can take the logarithm only of pure, dimensionless numbers, the proposed logarithmic transformation is best illustrated by an example. 
Step 1: Using the Bohart-Adams model as our example, its variables/parameters are assigned an appropriate set of units, e.g., $C_{\mathrm{o}}: \mathrm{mg} \mathrm{cm} \mathrm{cm}^{-3} ; t \mathrm{~min} ; L: \mathrm{cm} ; u: \mathrm{cm} \mathrm{min}^{-1} ; N_{\mathrm{o}}: \mathrm{mg} \mathrm{cm}^{-3}$; and $k_{\mathrm{BA}}: \mathrm{cm}^{3} \mathrm{mg}^{-}$ ${ }^{1} \min ^{-1}$.

Step 2: We define a new set of variables/parameters in the chosen system of units whose numerical values are equal to unity, e.g., $C_{\mathrm{o}}^{*}=1 \mathrm{mg} \mathrm{cm}^{-3} ; t^{*}=1 \mathrm{~min} ; L^{*}=1 \mathrm{~cm} ; u^{*}=1 \mathrm{~cm} \mathrm{~min}^{-1}$; and $N_{\mathrm{o}}^{*}=1 \mathrm{mg} \mathrm{cm}^{-3}$.

Step 3: The new variables/parameters are introduced into the Bohart-Adams model:

$$
\frac{C}{C_{\mathrm{o}}}=\frac{1}{1+\exp \left[\left(\frac{N_{\mathrm{o}}^{*} L^{*} u^{*}}{N_{\mathrm{o}}^{*} L^{*} u^{*}}\right)\left(\frac{k_{\mathrm{BA}} N_{\mathrm{o}} L}{u}\right)-\left(\frac{C_{\mathrm{o}}^{*} t^{*}}{C_{\mathrm{o}}^{*} t^{*}}\right)\left(k_{\mathrm{BA}} C_{\mathrm{o}} t\right)\right]}
$$

Note that the preceding equation is numerically identical to the original Bohart-Adams model.

Step 4: Upon rearrangement of the four bracketed terms within the exponential term in Eq. (12), we obtain

$$
\frac{C}{C_{\mathrm{o}}}=\frac{1}{1+\exp \left[\left(\frac{N_{\mathrm{o}}^{*} L^{*}}{u^{*}} k_{\mathrm{BA}}\right)\left(\frac{u^{*}}{N_{\mathrm{o}}^{*} L^{*}} \frac{N_{\mathrm{o}} L}{u}\right)-\left(C_{\mathrm{o}}^{*} t^{*} k_{\mathrm{BA}}\right)\left(\frac{1}{C_{\mathrm{o}}^{*} t^{*}} C_{\mathrm{o}} t\right)\right]}
$$

Step 5: We define two new parameters:

$\alpha=\frac{N_{\mathrm{o}}^{*} L^{*}}{u^{*}}, \operatorname{mg~min~} \mathrm{cm}^{-3} ; \beta=C_{\mathrm{o}}^{*} t^{*}, \mathrm{mg} \mathrm{min} \mathrm{cm}^{-3}$

Step 6: Substitution of Eq. (14) into Eq. (13) yields

$$
\frac{C}{C_{\mathrm{o}}}=\frac{1}{1+\exp \left[\left(\alpha k_{\mathrm{BA}}\right)\left(\frac{1}{\alpha} \frac{N_{\mathrm{o}} L}{u}\right)-\left(\beta k_{\mathrm{BA}}\right)\left(\frac{1}{\beta} C_{\mathrm{o}} t\right)\right]}
$$

All the four bracketed terms within the exponential term in Eq. (15) are dimensionless.

Step 7: Eq. (15) is empirically modified by taking the logarithm of the second and fourth bracketed terms within the exponential term:

$$
\frac{C}{C_{\mathrm{o}}}=\frac{1}{1+\exp \left[\left(\alpha k_{\mathrm{BA}}\right) \ln \left(\frac{1}{\alpha} \frac{N_{\mathrm{o}} L}{u}\right)-\left(\beta k_{\mathrm{BA}}\right) \ln \left(\frac{1}{\beta} C_{\mathrm{o}} t\right)\right]}
$$

Step 8: Because $\alpha$ and $\beta$ are numerically equal to unity, for convenience, these two parameters are omitted from Eq. (16):

$$
\frac{C}{C_{\mathrm{o}}}=\frac{1}{1+\exp \left[k_{\mathrm{BA}} \ln \left(\frac{N_{\mathrm{o}} L}{u}\right)-k_{\mathrm{BA}} \ln \left(C_{\mathrm{o}} t\right)\right]}
$$

The preceding equation is the new form of the Bohart-Adams model proposed in this work. It is referred to as the log-Bohart-Adams model. 
Because the Thomas and Yoon-Nelson models are analogous to the Bohart-Adams model, they can be transformed in the same spirit as the latter model. The resultant equations are

$$
\begin{aligned}
& \frac{C}{C_{\mathrm{o}}}=\frac{1}{1+\exp \left[k_{\mathrm{T}} \ln \left(\frac{q_{\mathrm{o}} M}{Q}\right)-k_{\mathrm{T}} \ln \left(C_{\mathrm{o}} t\right)\right]} \\
& \frac{C}{C_{\mathrm{o}}}=\frac{1}{1+\exp \left[k_{\mathrm{YN}} \ln (\tau)-k_{\mathrm{YN}} \ln (t)\right]}
\end{aligned}
$$

In this work, Eqs. (18) and (19) are known as the log-Thomas model and the log-Yoon-Nelson model, respectively. Just like the original models on which they are based, the log-Bohart-Adams, logThomas, and log-Yoon-Nelson models are equivalent to one another. Note that each of the three modified models remains a two-parameter model. Each expression contains no new parameters, but uses existing parameters and variables in new combinations. The three modified equations can be rearranged to give the following linear forms:

$$
\begin{aligned}
& \ln \left(\frac{C_{\mathrm{o}}}{C}-1\right)=k_{\mathrm{BA}} \ln \left(\frac{N_{\mathrm{o}} L}{u}\right)-k_{\mathrm{BA}} \ln \left(C_{\mathrm{o}} t\right) \\
& \ln \left(\frac{C_{\mathrm{o}}}{C}-1\right)=k_{\mathrm{T}} \ln \left(\frac{q_{\mathrm{o}} M}{Q}\right)-k_{\mathrm{T}} \ln \left(C_{\mathrm{o}} t\right) \\
& \ln \left(\frac{C_{\mathrm{o}}}{C}-1\right)=k_{\mathrm{YN}} \ln (\tau)-k_{\mathrm{YN}} \ln (t)
\end{aligned}
$$

A plot of the left-hand side of the three preceding equations versus $\ln \left(C_{\mathrm{o}} t\right)$ or $\ln (t)$ should result in a straight line if the data follow the linearized model.

\section{Nonlinear least-squares regression}

The modified models and their original counterparts are nonlinear functions with two fitting parameters. Nonlinear least-squares regression was applied to estimate their parameters. The overall fit of a model was measured with the coefficient of determination $R^{2}$. Although it should be well known that $R^{2}$ (adjusted or unadjusted) is not valid for nonlinear regression, it is still being used routinely within the environmental adsorption literature to assess the goodness of nonlinear fits. The most general definition of $R^{2}$ is given by

$$
R^{2}=1-\frac{\sum_{j=1}^{N}\left(\hat{z}_{j}-z_{j}\right)^{2}}{\sum_{j=1}^{N}\left(\hat{z}_{j}-\bar{z}\right)^{2}}
$$

where $N \equiv$ the number of data points; $\hat{z}_{j} \equiv$ model values; $z_{j} \equiv$ experimental values; and $\bar{z} \equiv$ the mean of experimental values. Given that $R^{2}$ can be misleading and inappropriate for discrimination of nonlinear models [27-29], it is supplemented here with a more general goodness-of-fit statistic known as the residual root mean square error (RRMSE) [30,31]: 
RRMSE $=\sqrt{\frac{\sum_{j=1}^{N}\left(\hat{z}_{j}-z_{j}\right)^{2}}{N-p}}$

where $p \equiv$ the number of regression parameters and $(N-p) \equiv$ the number of degrees of freedom of the regression. The RRMSE metric can be interpreted as the typical deviation of the data points from the fitted curve, and is valid for both linear and nonlinear regression models. Note that the RRMSE measure is also known as the standard error of the residuals [32]. A lower RRMSE value indicates a better fit.

\section{Results and discussion}

Three sets of skewed breakthrough data, selected from reports published in the literature of environmental adsorption, are used to test the three modified models. Because the modified models are analogous, it is not necessary to fit all three to the same set of breakthrough data. Accordingly, for a given set of breakthrough data, one of the three modified models will be selected for evaluation. The findings are applicable to the other two. The choice of which model to use is based on the type of information available. While most published modeling studies involving the Bohart-Adams and Thomas models provide information on the feed concentration $\left(C_{\mathrm{o}}\right)$ and flow rate $(u$ or $Q)$, many do not state the length of packed bed $(L)$ or mass of adsorbent $(M)$ used in the fixed bed experiments. So, if $L$ is available, we can select the log-Bohart-Adams model. If $M$ is given, we can pick the log-Thomas. If both $L$ and $M$ are available, either of the two models may be used. Because the log-Yoon-Nelson model does not contain typical system and operational variables, it is suitable for any chosen set of breakthrough data. The performance of a modified model is compared with that of the original version. Additional modeling results for another four sets of breakthrough data are provided in supplementary material, and these will be discussed later.

\subsection{Case 1: adsorption of fluoride by bone char}

This case reports the breakthrough characteristics of fluoride adsorption on two types of commercial bone char [33]. Fixed bed experiments were performed in a glass column with an internal diameter of $2.5 \mathrm{~cm}$ and a total length of $13 \mathrm{~cm}$. The column was packed with $27.69 \mathrm{~g}$ of bone char, resulting in a packed length of $7.5 \mathrm{~cm}$. For each bone char adsorbent, six breakthrough curves were measured using different flow rates and feed concentrations of fluoride. Tovar-Gómez et al. [33] found noticeable discrepancy between breakthrough data exhibiting tailing and prediction of the linearized Thomas model given by Eq. (4). To obtain better fits, they proposed the use of a hybrid neural networkThomas modeling approach.

Here, we have the option of choosing one out of the three modified models to correlate a set of breakthrough data taken from the work of Tovar-Gómez et al. [33]. We can select the log-BohartAdams model since the length of packed bed $(L)$ is available. With the mass of adsorbent $(M)$ given, the log-Thomas model is also a good choice. It is of course possible to pick the log-Yoon-Nelson model 
since it is free of standard system and operational variables. The log-Bohart-Adams model given by Eq. (17) was selected for this case study.

The chosen set of breakthrough data is shown in Fig. 1. The relevant experimental conditions are as follows: $C_{\mathrm{o}}=9 \times 10^{-3} \mathrm{mg} \mathrm{cm}^{-3}, u=0.67 \mathrm{~cm} \mathrm{~min}^{-1}$, and $L=7.5 \mathrm{~cm}$. The data points in the initial portion of the breakthrough curve indicate that fluoride breakthrough occurred almost immediately. A sharp rise of the dimensionless effluent concentration to about $70 \%$ was followed by a relatively slow approach to saturation, a characteristic of the tailing phenomenon which has been attributed to a variety of factors, including nonspecific adsorption, flow non-uniformity, heterogeneous particle size distribution, and so on. Accounting for these possible causes of tailing mechanistically requires the use of complex models.

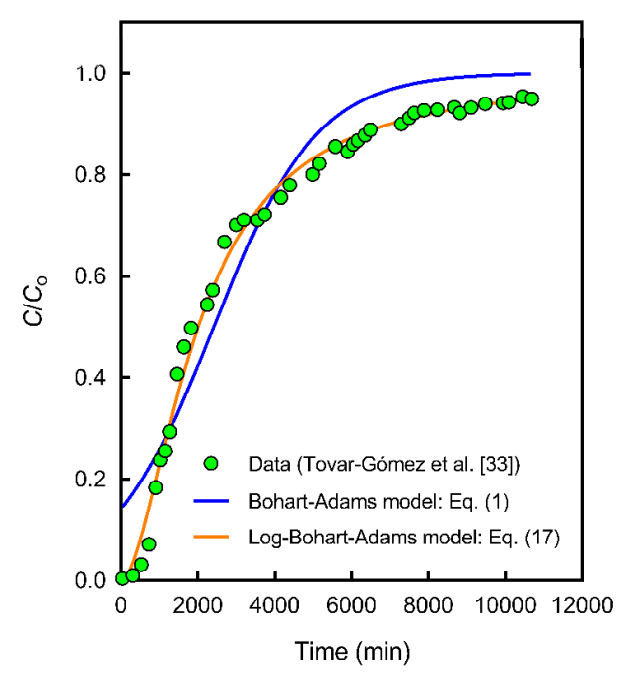

Fig. 1. Bohart-Adams and log-Bohart-Adams data fits, computed using nonlinear regression generated parameter estimates, compared to observed fluoride breakthrough data.

Fig. 1 shows the Bohart-Adams and log-Bohart-Adams data fits, computed from Eqs. (1) and (17) respectively using the best-fit parameters obtained from nonlinear regression analysis. Table 1 presents a summary of the parameter estimates and goodness-of-fit statistics for the two data fits. Fig. 1 illustrates vividly the inappropriateness of fitting the Bohart-Adams model to the asymmetrically distributed data. The Bohart-Adams data fit is highly unsatisfactory, displaying considerable overprediction of effluent concentrations in both the initial $\left(C / C_{0}<0.2\right)$ and later $\left(C / C_{0}>0.8\right)$ stages of fluoride breakthrough and underprediction of effluent concentrations in the middle region of the observed curve. In fact, the Bohart-Adams fitted curve missed most of the data points. By contrast, the $\log$-Bohart-Adams data fit shown in Fig. 1 is quite remarkable in its accuracy over the entire trajectory of the experimental data. This visual observation is well supported by the $R^{2}$ and RRMSE values presented in Table 1. It is apparent that the log-Bohart-Adams model is highly effective in correlating this set of asymmetric data. 
Table 1 Parameter estimates and goodness-of-fit statistics for the Bohart-Adams and log-Bohart-Adams data fits shown in Fig. 1.

\begin{tabular}{lllll}
\hline Model & $\begin{array}{l}N_{\mathrm{o}} \\
\left.(\mathrm{mg} \mathrm{cm})^{-3}\right)\end{array}$ & $\begin{array}{l}k_{\mathrm{BA}} \\
\left(\mathrm{cm}^{3} \mathrm{mg}^{-1} \mathrm{~min}^{-1}\right)\end{array}$ & $\begin{array}{l}R^{2} \\
(\%)\end{array}$ & RRMSE \\
\hline Bohart-Adams & 1.95 & 0.08 & 93.2 & 0.081 \\
Log-Bohart-Adams & 1.62 & 1.76 & 99.4 & 0.025 \\
\hline
\end{tabular}

At $t=0$, the Bohart-Adams equation predicted an effluent concentration percentage of $14.2 \%$. This relatively large effluent concentration contradicts the expected value of zero. The nonzero effluent concentration may be deduced from Eq. (1), which reduces to the following form when $t=0$ :

$$
\frac{C}{C_{\mathrm{o}}}=\frac{1}{1+\exp \left(\frac{k_{\mathrm{BA}} N_{\mathrm{o}} L}{u}\right)}
$$

This undesired attribute is due to the fact that the Bohart-Adams model is not valid for $t<\varepsilon L / u$ [34] where $\varepsilon$ is the bed voidage. It follows that the Bohart-Adams equation is undefined when $t=0$. The log-Bohart-Adams model is discernibly undefined when $t=0$ because the time variable $t$ has been replaced by the logarithmic term $\ln (t)$. Both the Bohart-Adams and log-Bohart-Adams models have the undesirable property of not being constrained to go through the 0,0 origin. However, the latter has the ability to predict very low effluent concentrations as $t$ approaches zero.

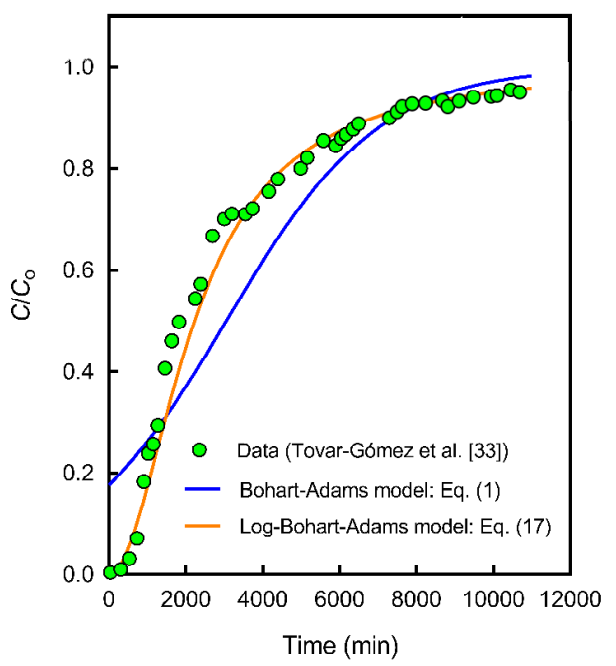

Fig. 2. Bohart-Adams and log-Bohart-Adams data fits, computed using linear regression generated parameter estimates, compared to observed fluoride breakthrough data.

Since both the Bohart-Adams and log-Bohart-Adams models have linear forms, it is of interest to know their relative performance. To answer this question the linear versions of the two models given by Eqs. (2) and (20) were fitted to the Fig. 1 data by linear regression, the details of which are not shown 
here for brevity (regression plots and parameter estimates). The Bohart-Adams linear fit with an $R^{2}$ score of $79.5 \%$ is very poor. In the case of the linearized log-Bohart-Adams the fit quality is reasonable $\left(R^{2}=98.1 \%\right)$. Fig. 2 plots the model curves computed from Eqs. (1) and (17) using the linear regression generated parameter estimates. For the log-Bohart-Adams model, the computed curve of Fig. 2 $($ RRMSE $=0.039)$ is slightly inferior to the data fit of Fig. 1 (RRMSE $=0.025)$. For the Bohart-Adams model, the computed curve of Fig. 2 (RRMSE $=0.11$ ) is worse than the data fit of Fig. 1 (RRMSE = 0.081). Clearly the linearized log-Bohart-Adams model is quantitatively superior to the linearized Bohart-Adams, and surprisingly quite similar to its nonlinear version.

From the perspective of overall fit, there is no question that the log-Bohart-Adams model is vastly superior to the original model form. However, the accuracy of fit is not an exclusively satisfactory criterion; the ability to predict reliable breakthrough and exhaustion times for randomly selected effluent concentrations also needs further consideration. This is because a good overall fit can sometimes mask inadequate fits to the initial and saturation regions of a breakthrough curve, where the breakthrough and exhaustion times reside. For the breakthrough concentration, it is common to select a data point in the close vicinity of $C / C_{\mathrm{o}}=10 \%$. For the saturation concentration, a data point in the immediate vicinity of $C / C_{\mathrm{o}}=90 \%$ is appropriate. Referring to Fig. 1, we see that it is not possible to pick a small effluent concentration as the breakthrough concentration because the Bohart-Adams fitted curve intersected the vertical axis at $C / C_{\mathrm{o}}=14.2 \%$ when $t=0$. The first data point larger than $14.2 \%$ was therefore selected as the breakthrough concentration, i.e., $C / C_{\mathrm{o}}=18.3 \%$ at $t=913 \mathrm{~min}$. The effluent concentration $C / C_{\mathrm{o}}=91.2 \%$ at $t=7494 \mathrm{~min}$ was chosen as the saturation concentration.

Rearranging the Bohart-Adams and log-Bohart-Adams models, we obtain

$$
\begin{aligned}
& t=\frac{1}{k_{\mathrm{BA}} C_{\mathrm{o}}}\left[\frac{k_{\mathrm{BA}} N_{\mathrm{o}} L}{u}-\ln \left(\frac{C_{\mathrm{o}}}{C}-1\right)\right] \\
& t=\frac{N_{\mathrm{o}} L}{\exp \left[\frac{1}{k_{\mathrm{BA}}} \ln \left(\frac{C_{\mathrm{o}}}{C}-1\right)\right] C_{\mathrm{o}} u}
\end{aligned}
$$

Given $C / C_{\mathrm{o}}$ values, Eqs. (26) and (27) yield time predictions for the Bohart-Adams and logBohart-Adams models, respectively. Breakthrough and exhaustion times corresponding to the selected effluent concentration levels can now be estimated from Eqs. (26) and (27). For case 1, the second column of Table 2 presents the observed and computed breakthrough and exhaustion times for the selected $C / C_{\mathrm{o}}$ values. The breakthrough time predicted by the log-Bohart-Adams model differs from the experimental one by $6 \%$, indicating that the early rising portion of the experimental breakthrough curve was well correlated by the model. In contrast, the Bohart-Adams prediction is markedly inferior to that of the log-Bohart-Adams, giving a huge error of $62.9 \%$. Comparison of the predicted exhaustion times indicates that the Bohart-Adams model is again inferior to the log-Bohart-Adams, with the former giving a discrepancy of $24.4 \%$ and the latter a trivial error of $1.2 \%$. Table 2 includes results for other cases and these will be discussed later. 
Table 2 Comparison of observed and predicted breakthrough and exhaustion times for selected effluent concentration levels.

\begin{tabular}{llll}
\hline Case & 1 & 2 & 3 \\
Model & Bohart-Adams & Thomas & Yoon-Nelson \\
\hline Experimental & & & \\
Breakthrough $C / C_{\mathrm{o}}(\%)$ & 18.3 & 10.4 & 11.5 \\
Breakthrough time (min) & 913 & 77 & 25 \\
Saturation $C / C_{\mathrm{o}}(\%)$ & 91.2 & 91.1 & 89.2 \\
Exhaustion time (min) & 7494 & 357 & 95 \\
Original model prediction & & & \\
Breakthrough time (min) & 339 & 41 & 19 \\
Error (\%) & 62.9 & 46.8 & 24 \\
Exhaustion time (min) & 5665 & 290 & 88 \\
Error (\%) & 24.4 & 18.8 & 7.4 \\
Modified model prediction & & & \\
Breakthrough time (min) & 858 & 68 & 24 \\
Error (\%) & 6 & 11.7 & 4 \\
Exhaustion time (min) & 7582 & 348 & 103 \\
Error (\%) & 1.2 & 2.5 & 8.4 \\
\hline
\end{tabular}

The physical significance of the fitted parameters given in Table 1 requires some discussion. The fitted $k_{\mathrm{BA}}$ value of the log-Bohart-Adams model is much bigger than that of the Bohart-Adams. This difference is due to the effect of the logarithmic transformation. As is well known, the BohartAdams rate formulation is based on a chemical reaction kinetics theory. Its single rate coefficient $k_{\mathrm{BA}}$ does not reflect the actual rate mechanism present in a typical liquid phase adsorption system, which is invariably controlled by mass transport processes. The parameter $k_{\mathrm{BA}}$ lacks physical significance and can thus be viewed as a purely empirical parameter which is adjusted to fit the shape of a given breakthrough curve. This consideration applies to both the Bohart-Adams and the log-Bohart-Adams models. The numerical value of $k_{\mathrm{BA}}$ does not in any way reflect the kinetics of the assumed chemical reaction.

In contrast to $k_{\mathrm{BA}}, N_{\mathrm{o}}$ is a physically significant parameter. This is because the Bohart-Adams model is derived from mass balance considerations. The numerical value of $N_{\mathrm{o}}$ indicates the adsorption capacity of the system under study. Strictly speaking, $N_{\mathrm{o}}$ for a fully saturated column should be invariant because the Bohart-Adams model assumes an irreversible adsorption isotherm. The amount of contaminant taken up by the adsorbent in a fixed bed is constant regardless of the feed concentration used and should correspond to the plateau of the rectangular adsorption isotherm. However, most actual 
systems do not exhibit irreversible adsorption equilibrium. In consequence, $N_{\mathrm{o}}$ is found to vary with the feed concentration.

Table 1 shows that the fitted $N_{\mathrm{o}}$ value of the Bohart-Adams model is $1.95 \mathrm{mg} \mathrm{cm}^{-3}$ for the case 1 adsorption system. The accuracy of this $N_{\mathrm{o}}$ value may be verified by comparing it to the experimental holdup capacity of the fixed bed, which is given by the area above the breakthrough curve and below the dimensionless effluent concentration of unity line. The total amount of fluoride retained by the fixed bed at saturation $(W)$ is given by the following expression:

$W=Q C_{\mathrm{o}}\left(t_{\mathrm{e}}-\int_{0}^{t_{\mathrm{e}}} \frac{C}{C_{\mathrm{o}}} d t\right)$

where $t_{\mathrm{e}} \equiv$ a time value at which $C / C_{\mathrm{o}}$ reaches unity or the breakthrough curve has leveled off. In Eq. (28), the quantity $W$ consists of two parts: the amount of fluoride taken up by the bone char adsorbent and the amount of fluoride remaining in the void volume of the fixed bed (empty space between the packing). Subtracting the amount of fluoride in the bed void volume from $W$ yields the amount of fluoride taken up by the adsorbent $\left(W_{\mathrm{a}}\right)$ :

$W_{\mathrm{a}}=Q C_{\mathrm{o}}\left(t_{\mathrm{e}}-\int_{0}^{t_{\mathrm{e}}} \frac{C}{C_{\mathrm{o}}} d t\right)-\varepsilon V_{\mathrm{b}} C_{\mathrm{o}}$

where $V_{\mathrm{b}} \equiv$ the bed volume.

According to Tovar-Gómez et al. [33], the void fraction ranged from $25 \%$ to $30 \%$ in all fixed bed adsorption experiments, giving an average $\mathcal{E}$ of $27.5 \%$. The remaining components of Eq. (29) are assigned the following values: $C_{\mathrm{o}}=9 \times 10^{-3} \mathrm{mg} \mathrm{cm}{ }^{-3}, Q=3.3 \mathrm{~cm}^{3} \mathrm{~min}^{-1}, V_{\mathrm{b}}=36.8 \mathrm{~cm}^{3}, t_{\mathrm{e}}$ is given by the final data point in Fig. 1 which is $10691 \mathrm{~min}$, and the integral, approximated by trapezoidal integration using the function trapz in MATLAB, is equal to 7707.7 min. Plugging these numbers into Eq. (29) spits out a $W_{\mathrm{a}}$ value of $88.51 \mathrm{mg}$. Dividing the $W_{\mathrm{a}}$ quantity by the bed volume $V_{\mathrm{b}}$ gives an uptake capacity of $2.41 \mathrm{mg} \mathrm{cm}^{-3}$. This quantity matches the definition of $N_{\mathrm{o}}$, which is equal to $1.95 \mathrm{mg}$ $\mathrm{cm}^{-3}$ according to the Bohart-Adams data fit. The fitted value of $N_{\mathrm{o}}$ is $19 \%$ smaller than the $W_{\mathrm{a}} / V_{\mathrm{b}}$ quantity. This rather small discrepancy is somewhat unexpected given the poor fit of the Bohart-Adams model, as may be seen in Fig. 1. The fairly close agreement between the $W_{\mathrm{a}} / V_{\mathrm{b}}$ quantity and $N_{\mathrm{o}}$ is probably fortuitous because the errors of overprediction and underprediction of the data fit practically cancelled each other out, reducing the overall error in $N_{\mathrm{o}}$. A good fit would likely lead to an increase in the $N_{\mathrm{o}}$ value, bringing it closer to the calculated $W_{\mathrm{a}} / V_{\mathrm{b}}$ quantity. The parameter $N_{\mathrm{o}}$ can then serve as a reliable indicator of the bed capacity. Unfortunately, the Bohart-Adams model is unable to track asymmetric breakthrough curves to a significant degree of accuracy. Note that the fixed bed test did not reach complete saturation. The effluent concentration of fluoride rose to only $95 \%$ of the feed solution concentration, that is, the final data point in Fig. 1 is given by $C / C_{\mathrm{o}}=0.95$. The actual $W_{\mathrm{a}} / V_{\mathrm{b}}$ quantity should thus be bigger than the estimated value of $2.41 \mathrm{mg} \mathrm{cm}^{-3}$. 
As can be seen in Table 1, the fitted $N_{\mathrm{o}}$ value of the log-Bohart-Adams model is $1.62 \mathrm{mg} \mathrm{cm}^{-3}$, which is $33 \%$ smaller than the $W_{\mathrm{a}} / V_{\mathrm{b}}$ quantity. Although the log-Bohart-Adams data fit is excellent, the logarithmic transformation distorted the fitted $N_{\mathrm{o}}$ value, making it unsuitable as a bed capacity indicator. Nevertheless, the main objective of the modification is to enhance the ability of the original BohartAdams model to fit asymmetric breakthrough curves, and to provide reliable estimates of breakthrough and exhaustion times. Accordingly, the fact that a less accurate $N_{\mathrm{o}}$ value has been obtained from the log-Bohart-Adams model is considered to be of secondary importance for our present concerns.

\subsection{Case 2: adsorption of methylene blue by activated carbon}

This example deals with dye removal by activated carbon synthesized from paper mill sewage sludge [35]. The carbon adsorbent was packed in a fixed bed column which was used to remove the dyes methylene blue and reactive red 24 from aqueous solutions. The column had an internal diameter of $1.2 \mathrm{~cm}$ and a total length of $20 \mathrm{~cm}$. The effects of influent dye concentration, particle size, adsorbent mass, and flow rate on the breakthrough characteristics of the two dyes were determined. Several sets of breakthrough curves are available in the article of Li et al. [35], many of which are asymmetric. Fig. 3 shows a set of methylene blue breakthrough data taken from their paper. In contrast to case 1, Fig. 3 shows that there is no immediate contaminant breakthrough in this case. The early portion of the experimental breakthrough curve is much shorter than the later stage, suggesting the presence of tailing.

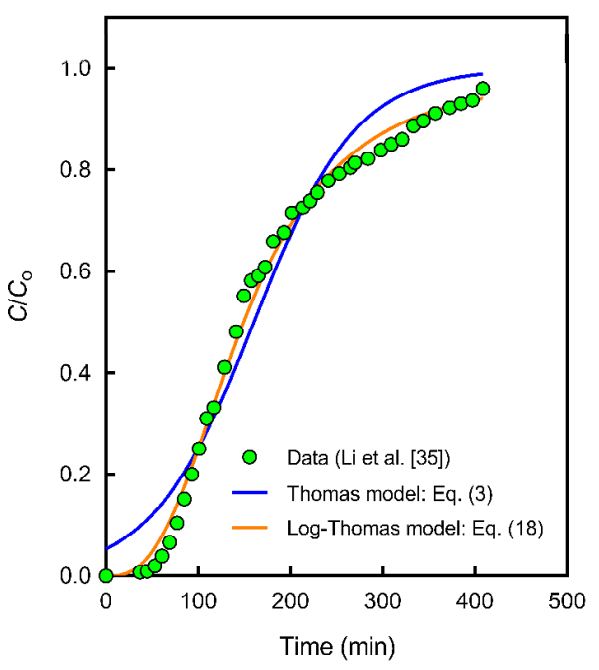

Fig. 3. Thomas and log-Thomas data fits compared to observed methylene blue breakthrough data.

The bed length parameter, $L$, for the selected fixed bed test is not available, ruling out the use of the Bohart-Adams and log-Bohart-Adams models to fit the Fig. 3 data. Since the mass of adsorbent is known, we can select the Thomas and log-Thomas models given by Eqs. (3) and (18) respectively to fit this data set. Their model input variables are as follows: $C_{\mathrm{o}}=0.1 \mathrm{mg} \mathrm{cm} \mathrm{cm}^{-3}, Q=5 \mathrm{~cm}^{3} \mathrm{~min}^{-1}$, and $M$ $=0.8$ g. Fig. 3 plots the two data fits, computed with the parameter estimates listed in Table 3. The Thomas data fit is rather poor, showing significant deviations at both the initial and later stages of the 
experimental curve, and displaying a fairly large effluent concentration at $t=0$. By contrast, it can be seen that the log-Thomas data fit is closely aligned with the entire experimental profile. The difference in the fit quality of the two models is also observable in the $R^{2}$ and RRMSE scores presented in Table 3. The log-Thomas model seems to work extremely well for this asymmetric data set.

Table 3 Parameter estimates and goodness-of-fit statistics for the Thomas and log-Thomas data fits shown in Fig. 3.

\begin{tabular}{lllll}
\hline Model & $\begin{array}{l}q_{\mathrm{o}} \\
\left(\mathrm{mg} \mathrm{g}^{-1}\right)\end{array}$ & $\begin{array}{l}k_{\mathrm{T}} \\
\left(\mathrm{cm}^{3} \mathrm{mg}^{-1} \mathrm{~min}^{-1}\right)\end{array}$ & $\begin{array}{l}R^{2} \\
(\%)\end{array}$ & RRMSE \\
\hline Thomas & 100.3 & 0.18 & 95.9 & 0.067 \\
Log-Thomas & 93.32 & 2.75 & 99.5 & 0.024 \\
\hline
\end{tabular}

The superiority of the log-Thomas model versus the original model is further demonstrated in predictions of breakthrough and exhaustion times. The third column of Table 2 presents the observed and predicted breakthrough and exhaustion times for the chosen effluent concentration levels. The breakthrough and exhaustion times predicted by the Thomas model differ from the corresponding observed values by $46.8 \%$ and $18.8 \%$, respectively. The log-Thomas model is much more accurate, yielding a discrepancy of $11.7 \%$ for the breakthrough time and an error of $2.5 \%$ for the exhaustion time. The log-Thomas model is undoubtedly the clear winner here.

The fitted $k_{\mathrm{T}}$ value of the log-Thomas model differs greatly from that of the Thomas model, as may be seen in Table 3. The logarithmic transformation turned $k_{\mathrm{T}}$ into a powerful shape parameter, producing the excellent log-Thomas data fit by optimizing the numerical value of $k_{\mathrm{T}}$. The Thomas rate coefficient $\left(k_{\mathrm{T}}\right)$, just like the Bohart-Adams rate coefficient $\left(k_{\mathrm{BA}}\right)$, can be viewed as a purely empirical parameter. This is because the Thomas model is based on an unrealistic surface reaction mechanism. In most cases mass transport processes control the kinetics of adsorption in porous activated carbon particles.

The Thomas capacity parameter $\left(q_{\mathrm{o}}\right)$, just like the Bohart-Adams capacity parameter $\left(N_{\mathrm{o}}\right)$, is physically significant because the Thomas model is based on mass conservation laws. It should be noted that Eq. (3) is a simplified form of the original model derived by Thomas. In Thomas's modeling approach, the value of $q_{\mathrm{o}}$ is given by the maximum adsorption capacity parameter of the Langmuir isotherm [2]. This condition is however ignored in many studies that use the simplified Thomas model in data fitting. The prevailing practice is to extract $q_{\mathrm{o}}$ from breakthrough data without regard to the type of equilibrium relationship of the adsorbate-adsorbent system under study. Table 3 shows that the $q_{\text {o }}$ value of the Thomas model obtained by this modeling approach is $100.3 \mathrm{mg} \mathrm{g}^{-1}$.

As mentioned previously, the area above the breakthrough curve gives the amount of contaminant adsorbed. Since the void fraction is not reported, we will use Eq. (28) in lieu of Eq. (29) to estimate the total amount of methylene blue retained by the fixed bed at saturation. The values of $C_{\mathrm{o}}$ 
and $Q$ required by Eq. (28) are given above. The final data point in Fig. 3 gives $t_{\mathrm{e}}=409 \mathrm{~min}$ and the integral in Eq. (28) is estimated as $232.7 \mathrm{~min}$. With these numbers, Eq. (28) works out a $W$ value of $88.2 \mathrm{mg}$. Dividing the $W$ quantity by the mass of adsorbent $M$ gives an uptake capacity of $110.2 \mathrm{mg} \mathrm{g}^{-}$

${ }^{1}$. There is good agreement between the fitted $q_{\mathrm{o}}$ value of the Thomas model $\left(100.3 \mathrm{mg} \mathrm{g}^{-1}\right)$ and the W/M quantity even though the data fit is not very accurate. As discussed in case 1 , the good agreement is probably fortuitous given the rather poor Thomas data fit. The fitted $q_{\mathrm{o}}$ value of the log-Thomas model $\left(93.32 \mathrm{mg} \mathrm{g}^{-1}\right)$ is surprisingly quite comparable to the $W / M$ quantity of $110.2 \mathrm{mg} \mathrm{g}^{-1}$. It seems that in this case the distortion effect of the logarithmic transformation on $q_{\mathrm{o}}$ was not significant.

\subsection{Case 3: adsorption of salicylic acid by imprinted adsorbent}

This third case describes breakthrough results for salicylic acid adsorption on a wollastonitebased imprinted adsorbent [36]. A glass column with an internal diameter of $1 \mathrm{~cm}$ and a length of 30 $\mathrm{cm}$ (packed length $=0.9-4.6 \mathrm{~cm}$ ) was used to produce a series of breakthrough curves as functions of initial adsorbate concentration, flow rate, bed depth, and temperature. A set of breakthrough data taken from the work of Meng et al. [36] is presented in Fig. 4. The experimental conditions for this fixed bed test are as follows: $C_{\mathrm{o}}=0.01 \mathrm{mg} \mathrm{cm}^{-3}, Q=1 \mathrm{~cm}^{3} \mathrm{~min}^{-1}$, and $L=0.9 \mathrm{~cm}$. Fig. 4 shows that salicylic acid emerged fairly quickly at the column outlet.

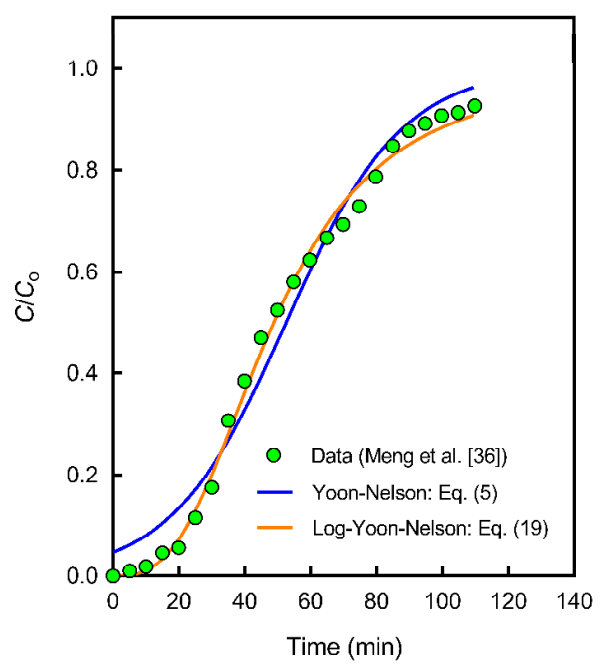

Fig. 4. Yoon-Nelson and log-Yoon-Nelson data fits compared to observed salicylic acid breakthrough data.

Since the original and modified Bohart-Adams and Thomas models have been tested in the previous two cases, for this example the only ones left to be tested are the Yoon-Nelson model given by Eq. (5) and the log-Yoon-Nelson given by Eq. (19). As noted previously, use of the Yoon-Nelson model and its logarithmic variant in data fitting is straightforward because they lack typical system and operational variables. Table 4 presents the regression results while Fig. 4 plots the Yoon-Nelson and $\log$-Yoon-Nelson data fits. 
Table 4 Parameter estimates and goodness-of-fit statistics for the Yoon-Nelson and log-Yoon-Nelson data fits shown in Fig. 4.

\begin{tabular}{lllll}
\hline Model & $\tau$ & $k_{\mathrm{YN}}$ & $R^{2}$ & RRMSE \\
& $(\mathrm{min})$ & $\left(\mathrm{min}^{-1}\right)$ & $\begin{array}{l}(\%) \\
\end{array}$ \\
\hline Yoon-Nelson & 52.62 & 0.06 & 98.0 & 0.049 \\
Log-Yoon-Nelson & 48.85 & 2.84 & 99.6 & 0.023 \\
\hline
\end{tabular}

The Yoon-Nelson data fit is somewhat decent, especially around the saturation portion of the breakthrough curve. There is however noticeable deviation in the early stage of salicylic acid breakthrough. In addition, there is an obvious nonzero effluent concentration at $t=0$ even though the observed breakthrough curve is not highly asymmetric. From the Fig. 4 plot, one can see that the logYoon-Nelson model is very effective, tracing the majority of the data points. However, the fact that the log-Yoon-Nelson model does not contain important fixed bed variables such as bed length makes it unsuitable as a design tool for sizing fixed bed adsorption columns.

The fourth column of Table 2 compares the relative performance of the two models in predicting breakthrough and exhaustion times corresponding to the selected effluent concentration levels. As expected, the breakthrough time predicted by the log-Yoon-Nelson model is quantitatively reasonable (error $=4 \%$ ). Because of the inability of the Yoon-Nelson model to fit the initial portion of the experimental curve, the error of its breakthrough time prediction is quite large $(24 \%)$. The exhaustion time predicted by the Yoon-Nelson model is marginally more accurate than the prediction of the log-Yoon-Nelson.

\subsection{Additional cases}

The Bohart-Adams and log-Bohart-Adams models were tested against another four sets of breakthrough data taken from reports published in the Journal of Water Process Engineering. The modeling results of these four cases, designated by S1-S4, are presented in supplementary material. Case S1 deals with the fixed bed adsorption of lead by an adsorbent based on waterworks sludge [37]. Case S2 illustrates the breakthrough behavior of mercury adsorption on a nano-adsorbent [38]. Case S3 reports breakthrough results for nickel sequestration by a seaweed biosorbent [39]. Case S4 describes the arsenic removal by a nano-adsorbent in fixed bed columns [40]. The results of all four cases, presented in Figs. S1-S4 and Tables S1-S4, indicate that the better fitting model is the log-BohartAdams. These results, taken together with those of cases 1-3, confirm that the modified models are very flexible and much more effective than the original versions in correlating asymmetric breakthrough data.

\subsection{What's in a name?}

The ability of the log-Bohart-Adams, log-Thomas, and log-Yoon-Nelson models to correlate asymmetric breakthrough data is mainly due to their superior functional form. To dig deeper into their 
functional form, it is necessary to rearrange the model equations defined by Eqs. (17)-(19). Using the log-Bohart-Adams as example, Eq. (17) may be rewritten as

$$
\frac{C}{C_{\mathrm{o}}}=\frac{1}{1+\frac{\left(N_{\mathrm{o}} L / u\right)^{k_{\mathrm{BA}}}}{\left(C_{\mathrm{o}} t\right)^{k_{\mathrm{BA}}}}}
$$

which may in turn be expressed as

$$
\frac{C}{C_{\mathrm{o}}}=\frac{1}{1+\left(\frac{N_{\mathrm{o}} L}{C_{\mathrm{o}} u}\right)^{k_{\mathrm{BA}}} t^{-k_{\mathrm{BA}}}}
$$

The general form of the preceding equation is given in Eq. (32).

$$
\frac{C}{C_{\mathrm{o}}}=\frac{1}{1+m t^{-n}}
$$

where

$$
\begin{aligned}
& \text { Log-Bohart-Adams: } m=\left(\frac{N_{\mathrm{o}} L}{C_{\mathrm{o}} u}\right)^{k_{\mathrm{BA}}} ; n=k_{\mathrm{BA}} \\
& \text { Log-Thomas : } m=\left(\frac{q_{\mathrm{o}} M}{C_{\mathrm{o}} Q}\right)^{k_{\mathrm{T}}} ; n=k_{\mathrm{T}} \\
& \text { Log-Yoon-Nelson : } m=\tau^{k_{\mathrm{YN}}} ; n=k_{\mathrm{YN}}
\end{aligned}
$$

Eq. (32), which predicts a sigmoid curve when $n>1$, is analogous to several mathematical models with different names. One such model was put forward by Hill [41] more than one hundred years ago. Hill, a mathematician turned physiologist, devised what is now known as the Hill equation to model oxygen binding to hemoglobin:

$$
\theta=\frac{m_{1} x^{n_{1}}}{1+m_{1} x^{n_{1}}}
$$

where $\theta \equiv$ the fractional binding or fractional response; $x \equiv$ the free ligand concentration; and $m_{1}, n_{1} \equiv$ constants. Eq. (36) may be rewritten as

$$
\theta=\frac{1}{1+\left(1 / m_{1}\right) x^{-n_{1}}}
$$

which is analogous to Eq. (32), with different constants.

Although the Hill equation has become ingrained in the field of quantitative pharmacology-especially for analysis of dose-response curves-it is treated as an empirical approximation.

To model asymmetric breakthrough curves, Yan et al. [20] put forward the following doseresponse model without claiming origins in the Hill formulation:

$$
\frac{C}{C_{\mathrm{o}}}=1-\frac{1}{1+\left(V / m_{2}\right)^{n_{2}}}
$$


where $V \equiv$ the throughput volume and $m_{2}, n_{2} \equiv$ constants. Eq. (38) may be rewritten as

$$
\frac{C}{C_{0}}=1-\frac{1}{1+\left(V / m_{2}\right)^{n_{2}}}=\frac{\left(V / m_{2}\right)^{n_{2}}}{1+\left(V / m_{2}\right)^{n_{2}}}=\frac{1}{1+m_{2}^{n_{2}} V^{-n_{2}}}
$$

The preceding equation reveals that the Yan model given by Eq. (38) is merely a rearranged form of the Hill equation. It follows that the Yan model is analogous to Eq. (32). To reduce the empirical nature of their dose-response model, Yan et al. [20] replaced the constant $m_{2}$ with some parameters of the Thomas model $\left(q_{\mathrm{o}}, M, C_{\mathrm{o}}\right.$ ) but they failed to incorporate the Thomas rate coefficient $k_{\mathrm{T}}$. Their hybrid model retains the empirical exponent $n_{2}$, a remnant of Hill's equation. By contrast, it is straightforward to convert the Thomas model to the mathematical form of Eq. (32) using the logarithmic transformation method outlined previously. The transformation works directly on the Thomas model, obviating the need for foreign parameters. The resultant model, the log-Thomas given by Eq. (18), contains nothing but the original parameters of the Thomas model and uses the Thomas rate coefficient $k_{\mathrm{T}}$ as the exponent.

Yet another dose-response model, known as the log-logistic equation, is equivalent to Eq. (32). This equation can be derived by applying the logarithmic transformation method to the logistic model defined by Eq. (7). Referring to Eq. (7), if we replace $a$ by $\ln (a)$ and $t$ by $\ln (t)$ (assuming $t$ has been made dimensionless), then we obtain

$\frac{C}{C_{\mathrm{o}}}=\frac{1}{1+\exp [\ln (a)-b \ln (t)]}$

which may be rewritten as

$$
\frac{C}{C_{\mathrm{o}}}=\frac{1}{1+a t^{-b}}
$$

Comparison of this last equation and Eq. (32) confirms that they are equivalent, with different constants.

Additionally, a growth curve model attributed to Hossfeld [42] is a direct analog of Eq. (32). This model, known as the Hossfeld IV equation, has been devised for the description of tree growth:

$$
\frac{y}{y_{\max }}=\frac{1}{1+m_{3} t^{-n_{3}}}
$$

where $y \equiv$ the response variable; $y_{\max } \equiv$ the maximum value of $y$; and $m_{3}, n_{3} \equiv$ constants. Evidently, the Hossfeld IV model, originally proposed in 1822 and therefore much older than the Hill equation, is functionally identical with Eq. (32).

The Sips adsorption isotherm [43], also known as the Langmuir-Freundlich isotherm, is well known for its ability to model a wide variety of equilibrium data. It bears an obvious resemblance to the Hill equation, and can therefore be expressed in the functional form of Eq. (32). The Sips isotherm is given by 
$\frac{q}{q_{\max }}=\frac{m_{4} c^{n_{4}}}{1+m_{4} c^{n_{4}}}$

where $q \equiv$ the solid phase contaminant concentration; $q_{\max } \equiv$ the maximum adsorption capacity; $c \equiv$ the fluid phase concentration; and $m_{4}, n_{4} \equiv$ constants. Eq. (43) may be rewritten as

$$
\frac{q}{q_{\max }}=\frac{1}{1+\left(1 / m_{4}\right) C^{-n_{4}}}
$$

When $n_{4}<1$, the Sips isotherm yields a portion of a rectangular hyperbola, and when $n_{4}>1$ it produces a sigmoid curve. When $n_{4}=1$, the Sips isotherm reduces to the Langmuir isotherm. Interestingly, in 1909 Hill [44] put forward what later became known as Langmuir's adsorption isotherm. Langmuir [45]—seemingly without knowledge of the prior work of Hill—published his namesake isotherm equation a few years later. In some publications, the eponym "Hill-Langmuir" is used to denote Langmuir's hyperbolic relation.

We see from the foregoing discussion Eq. (32) stretches out its tentacles to a wide variety of curve-fitting models. Like the novel coronavirus responsible for COVID-19, Eq. (32) has the congenital ability to latch onto ever more new targets: the Bohart-Adams, Thomas, and Yoon-Nelson models have now been infected. Eq. (32) has one functional form but several names, including log-Bohart-Adams, log-Thomas, log-Yoon-Nelson, Hill, Yan, log-logistic, Hossfeld IV, Sips, and Langmuir-Freundlich. This name list is by no means exhaustive, but it should help demonstrate the ubiquitous influence of Eq. (32) in the modeling of sigmoid as well as hyperbolic curves. In cases 1-3 and S1-S4, we have demonstrated the ability of Eq. (32) to accurately correlate asymmetric breakthrough data. The next section explores a fundamental property of Eq. (32)—point of inflection—on which its data fitting ability depends.

\subsection{Inflection points}

A sigmoid curve is characterized by the presence of an inflection point, at which the curvature changes from concave to convex or vice versa. For a fixed bed model capable of exhibiting sigmoid curves, the location of the inflection point can be computed in two simple steps. First, the time value at the inflection point may be estimated by setting the second derivative of the model equation to zero. Next, substituting the derived time value in the model equation yields the response value at the inflection point, which, for a breakthrough curve model, corresponds to the $C / C_{\mathrm{o}}$ value at the inflection point. The symmetry of a sigmoid breakthrough curve is dictated by the location of its inflection point. A breakthrough curve is symmetric if its inflection point corresponds to the midpoint, i.e., $C / C_{\mathrm{o}}=0.5$, and asymmetric if it does not.

For the Bohart-Adams, Thomas, and Yoon-Nelson models, which are fully equivalent to the logistic equation, their inflection points match the midpoint $\left(C / C_{\mathrm{o}}=0.5\right)$ irrespective of the values of their model parameters. With this invariant inflection point, the three widely used models of fixed bed adsorption are inherently symmetric. As such, they yielded the symmetric curves shown in cases 1-3, 
which failed to track the asymmetric profiles of the experimental data and provided incorrect estimates of breakthrough and exhaustion times.

For the log-Bohart-Adams, log-Thomas, and log-Yoon-Nelson models, which are represented by Eqs. (32)-(35), the location of the inflection point is given by

$\frac{C}{C_{\mathrm{o}}}=\frac{n-1}{2 n}$

where $n>1$ and $n=k_{\mathrm{BA}}, k_{\mathrm{T}}, k_{\mathrm{YN}}$ (these rate coefficients are treated as dimensionless quantities, see Eqs. (16) and (17)). The inflection point is a function of $n$; it is greater than zero and the upper limit is 0.5 . The inflection points for the breakthrough curves predicted by the three modified models for cases 13, computed from Eq. (45) with the fitted values of $k_{\mathrm{BA}}, k_{\mathrm{T}}$, and $k_{\mathrm{YN}}$, are presented in Table 5. Mathematically, the three modified models possess two distinct characteristics—a floating inflection point and a flexible functional form - that together enhance their ability to accurately correlate the asymmetric breakthrough data of cases $1-3$, and to afford reliable estimates of breakthrough and exhaustion times (see Table 2).

Table 5 Inflection points for the breakthrough curves predicted by the log-Bohart-Adams, log-Thomas, and log-Yoon-Nelson models (see Figs. 1, 3, and 4).

\begin{tabular}{llll}
\hline Case & Model & $n$ & Inflection point $\left(C / C_{\mathrm{o}}\right)$ \\
\hline 1 & Log-Bohart-Adams & 1.76 & 0.216 \\
2 & Log-Thomas & 2.75 & 0.318 \\
3 & Log-Yoon-Nelson & 2.84 & 0.324 \\
\hline
\end{tabular}

The trend of the inflection points for the three cases may be used to gauge the extent of curve asymmetry, as shown in the top plot of Fig. 5. Case 1 has the smallest inflection point, one may thus surmise that the further the inflection point drifts from the mid-point, the stronger the curve asymmetry. This hypothesis is supported by the RRMSE trends for the original and modified model fits, as shown in the middle and bottom plots of Fig. 5. The RRMSE value for the original model fit for case 1 is the largest, indicating that the case 1 data set posed the hardest challenge to the original model, which is by design symmetric. One may infer that case 1 must have displayed the strongest asymmetry among the three cases. The RRMSE value for the modified model fit for case 1 may be interpreted in the same way-it is the biggest, and so the case 1 data set was the most difficult to fit, implying that case 1 must have exhibited the strongest asymmetry. It should be recalled that the three modified models are analogous to one another; so are the three original versions. The comparison of the three cases in Fig. 5 is therefore valid although each case was analyzed using a particular modified model and its original form. 

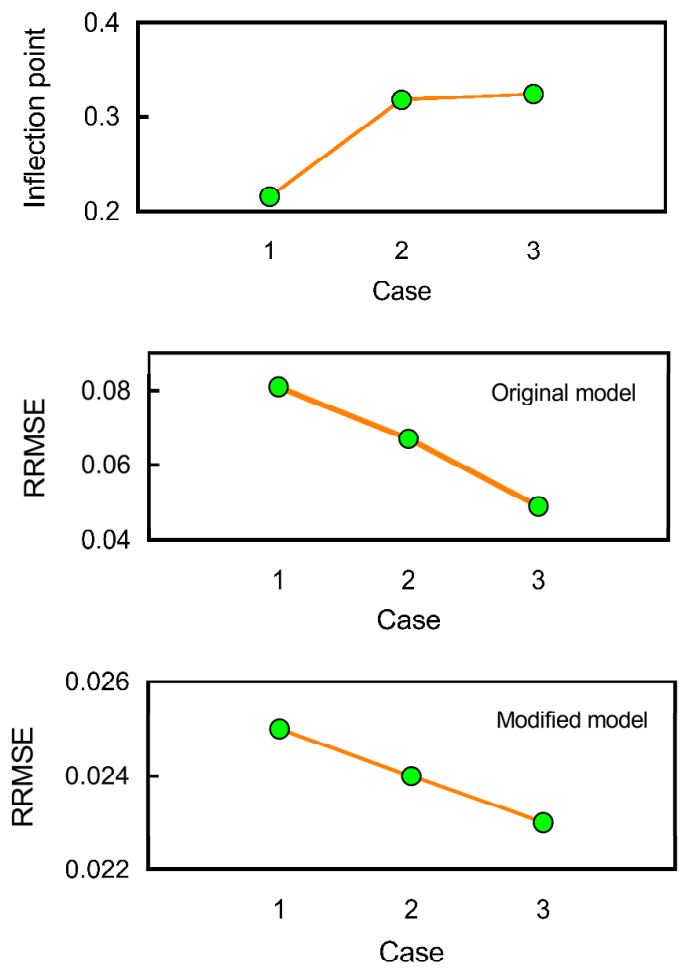

Fig. 5. Trends of inflection points and RRMSE values.

\section{Conclusions}

The three modified models have been shown to successfully represent the asymmetric breakthrough curves for the adsorption of seven different water contaminants in fixed bed columns packed with adsorbents of different types. Consequently, they were found capable of providing reliable estimates of breakthrough and exhaustion times corresponding to any predetermined concentration level. Thus, their practical value is evident. In all the cases studied, the three original models were found to perform poorly, resulting in inaccurate estimates of breakthrough and exhaustion times. However, the fitted capacity parameter of the modified Bohart-Adams model was found somewhat inaccurate. This shortcoming is of secondary importance because the practical utility of the modified BohartAdams model is estimation of breakthrough and exhaustion times.

A unique feature of the modified models is that they contain no new parameters, which is one of the key points of this paper. Existing models capable of describing asymmetric breakthrough curves invariably rely on extra shape parameters. Although the modified models are highly flexible, being capable of assuming a great variety of different sigmoid shapes, it could be argued that their general applicability cannot be inferred from the seven cases tested here. It is likely that contaminant adsorption on synthetic and natural adsorbents of different types will produce a myriad of breakthrough curve shapes. Additional testing using experimental breakthrough data of other water contaminants such as 
micropollutants will be required to give confidence that the modified models may be universally applied.

\section{Funding}

This work was not in any way directly or indirectly supported, funded, or sponsored by any organization or entity.

\section{Acknowledgments}

The authors would like to acknowledge the use of the breakthrough data previously published by Dr. R. Tovar-Gómez, Dr. Wenhong Li, Dr. Minjia Meng, Dr. Ayad A.H. Faisal, Dr. Seyedeh Sima Ghasemi, Dr. C.E.R. Barquilha, Dr. Sara Contreras, and their collaborators. Several recent articles [4649] exposing the questionable practice of citation coercion by rogue reviewers proved useful during the peer review process. Say NO to citation coercion. The authors thank the handling editor, Dr. Guangming Jiang, for his support and encouragement.

\section{Declaration of competing interest}

The authors declare that they have no known competing financial interests or personal relationships that could have appeared to influence the work reported in this paper.

\section{References}

[1] G.S. Bohart, E.Q. Adams, Some aspects of the behavior of charcoal with respect to chlorine, J. Am. Chem. Soc. 42 (1920) 523-544.

[2] H.C. Thomas, Heterogeneous ion exchange in a flowing system, J. Am. Chem. Soc. 66 (1944) 1664-1666.

[3] Y.H. Yoon, J.A. Nelson, Application of gas adsorption kinetics. I. A theoretical model for respirator cartridge service life, Am. Ind. Hyg. Assoc. J. 45 (1984) 509-516.

[4] V. Díaz-Blancas, C.G. Aguilar-Madera, J.V. Flores-Cano, R. Leyva-Ramos, E. Padilla-Ortega, R. Ocampo-Pérez, Evaluation of mass transfer mechanisms involved during the adsorption of metronidazole on granular activated carbon in fixed bed column, J. Water Process Eng. 36 (2020) 101303.

[5] D.S.P. Franco, J.L.S. Fagundes, J. Georgin, N.P.G. Salau, G.L. Dotto, A mass transfer study considering intraparticle diffusion and axial dispersion for fixed-bed adsorption of crystal violet on pecan pericarp (Carya illinoensis), Chem. Eng. J. 397 (2020) 125423.

[6] W.J. Weber Jr, E.H. Smith, Simulation and design models for adsorption processes, Environ. Sci. Technol. 21 (1987) 1040-1050.

[7] E. Worch, Fixed-bed adsorption in drinking water treatment: a critical review on models and parameter estimation, J. Water Supply Res. Technol. AQUA 57 (2008) 171-183. 
[8] Z. Xu, J. Cai, B. Pan, Mathematically modeling fixed-bed adsorption in aqueous systems, J. Zhejiang Univ. Sci. A 14 (2013) 155-176.

[9] K.L. Tan, B.H. Hameed, Insight into the adsorption kinetics models for the removal of contaminants from aqueous solutions, J. Taiwan Inst. Chem. Eng. 74 (2017) 25-48.

[10] D.O. Cooney, Adsorption Design for Wastewater Treatment, Lewis Publishers, Boca Raton, 1999.

[11] C. Tien, Introduction to Adsorption: Basics, Analysis, and Applications, Elsevier, Amsterdam, 2019.

[12] K. Nithya, A. Sathish, P.S. Kumar, Packed bed column optimization and modeling studies for removal of chromium ions using chemically modified Lantana camara adsorbent, J. Water Process Eng. 33 (2020) 101069.

[13] E. Nishikawa, S.L. Cardoso, C.S.D. Costa, M.G.C. da Silva, M.G.A. Vieira, New perception of the continuous biosorption of cadmium on a seaweed derivative waste, J. Water Process Eng. 36 (2020) 101322.

[14] A. Chatterjee, S. Schiewer, Biosorption of cadmium(II) ions by citrus peels in a packed bed column: effect of process parameters and comparison of different breakthrough curve models, Clean-Soil Air Water 39 (2011) 874-881.

[15] C.-G. Lee, J.-H. Kim, J.-K. Kang, S.-B. Kim, S.-J. Park, S.-H. Lee, J.-W. Choi, Comparative analysis of fixed-bed sorption models using phosphate breakthrough curves in slag filter media, Desalin. Water Treat. 55 (2015) 1795-1805.

[16] K.H. Chu, Breakthrough curve analysis by simplistic models of fixed bed adsorption: in defense of the century-old Bohart-Adams model, Chem. Eng. J. 380 (2020) 122513.

[17] R.M. Clark, J.M. Symons, J.C. Ireland, Evaluating field scale GAC systems for drinking water, J. Environ. Eng. 112 (1986) 744-756.

[18] R.M. Clark, Modeling TOC removal by GAC: the general logistic function, J. Am. Water Works Assn. 79 (1987) 33-37.

[19] R.M. Clark, Evaluating the cost and performance of field-scale granular activated carbon systems, Environ. Sci. Technol. 21 (1987) 573-580.

[20] G. Yan, T. Viraraghavan, M. Chen, A new model for heavy metal removal in a biosorption column, Adsorp. Sci. Technol. 19 (2001) 25-43.

[21] N. Blagojev, D. Kukić, V. Vasić, M. Šćiban, J. Prodanović, O. Bera, A new approach for modelling and optimization of $\mathrm{Cu}(\mathrm{II})$ biosorption from aqueous solutions using sugar beet shreds in a fixed-bed column, J. Hazard. Mater. 363 (2019) 366-375.

[22] Q. Hu, Y. Xie, C. Feng, Z. Zhang, Fractal-like kinetics of adsorption on heterogeneous surfaces in the fixed-bed column, Chem. Eng. J. 358 (2019) 1471-1478.

[23] W.W. Eckenfelder Jr., Industrial Water Pollution Control, McGraw-Hill, Boston, 1967. 
[24] W.W. Eckenfelder, D.L. Ford, Water Pollution Control: Experimental Procedures for Process Design, Pemberton Press, Austin, 1970.

[25] H.C. Thomas, Chromatography: a problem in kinetics, Ann. N. Y. Acad. Sci. 49 (1948) 161168.

[26] T.D. Reynolds, Unit Operations and Processes in Environmental Engineering, Wadsworth, Belmont, 1982.

[27] T.O. Kvålseth, Cautionary note about $R^{2}$, Am. Stat. 39 (1985) 279-285.

[28] A.-N. Spiess, N. Neumeyer, An evaluation of $\mathrm{R}^{2}$ as an inadequate measure for nonlinear models in pharmacological and biochemical research: a Monte Carlo approach, BMC Pharmacol. 10 (2010) 6.

[29] J. Tellinghuisen, C.H. Bolster, Using $R^{2}$ to compare least-squares fit models: when it must fail, Chemometr. Intell. Lab. Syst. 105 (2011) 220-222.

[30] D.G. Kinniburgh, General purpose adsorption isotherms, Environ. Sci. Technol. 20 (1986) 895904.

[31] A. Kapoor, R.T. Yang, Correlation of equilibrium adsorption data of condensible vapours on porous adsorbents, Gas Sep. Purif. 3 (1989) 187-192.

[32] A.M. Brown, A step-by-step guide to non-linear regression analysis of experimental data using a Microsoft Excel spreadsheet, Comput. Methods Programs Biomed. 65 (2001) 191-200.

[33] R. Tovar-Gómez, M.R. Moreno-Virgen, J.A. Dena-Aguilar, V. Hernández-Montoya, A. BonillaPetriciolet, M.A. Montes-Morán, Modeling of fixed-bed adsorption of fluoride on bone char using a hybrid neural network approach, Chem. Eng. J. 228 (2013) 1098-1109.

[34] N.R. Amundson, A note on the mathematics of adsorption in beds, J. Phys. Colloid Chem. 52 (1948) 1153-1157.

[35] W. Li, Q. Yue, P. Tu, Z. Ma, B. Gao, J. Li, X. Xu, Adsorption characteristics of dyes in columns of activated carbon prepared from paper mill sewage sludge, Chem. Eng. J. 178 (2011) 197-203.

[36] M. Meng, Y. Feng, M. Zhang, Y. Liu, Y. Ji, J. Wang, Y. Wu, Y. Yan, Highly efficient adsorption of salicylic acid from aqueous solution by wollastonite-based imprinted adsorbent: a fixed-bed column study, Chem. Eng. J. 225 (2013) 331-339.

[37] A.A.H. Faisal, S.F.A. Al-Wakel, H.A. Assi, L.A. Naji, Mu. Naushad, Waterworks sludge-filter sand permeable reactive barrier for removal of toxic lead ions from contaminated groundwater, J. Water Process Eng. 33 (2020) 101112.

[38] S.S. Ghasemi, M. Hadavifar, B. Maleki, E. Mohammadnia, Adsorption of mercury ions from synthetic aqueous solution using polydopamine decorated SWCNTs, J. Water Process Eng. 32 (2019) 100965.

[39] C.E.R. Barquilha, E.S. Cossich, C.R.G. Tavares, E.A. da Silva, Biosorption of nickel and copper ions from synthetic solution and electroplating effluent using fixed bed column of immobilized brown algae, J. Water Process Eng. 32 (2019) 100904. 
[40] S. Contreras, L. Henríquez-Vargas, P.I. Álvarez, Arsenic transport and adsorption modeling in columns using a copper nanoparticles composite, J. Water Process Eng. 19 (2017) 212-219.

[41] A.V. Hill, The possible effects of the aggregation of the molecules of haemoglobin on its dissociation curve, J. Physiol. 40 (1910) iv-vii.

[42] J.W. Hossfeld, Mathematik für Forstmänner, Ökonomen und Cameralisten, Vol. 4. Hennings, Gotha, 1822.

[43] R. Sips, On the structure of a catalyst surface, J. Chem. Phys. 16 (1948) 490-495.

[44] A.V. Hill, The mode of action of nicotine and curari, determined by the form of the contraction curve and the method of temperature coefficients, J. Physiol. 39 (1909) 361-373.

[45] I. Langmuir, The constitution and fundamental properties of solids and liquids. Part I. Solids, J. Am. Chem. Soc. 38 (1916) 2221-2295.

[46] J.D. Wren, A. Valencia, J. Kelso, Reviewer-coerced citation: case report, update on journal policy and suggestions for future prevention, Bioinformatics 35 (2019) 3217-3218.

[47] D. Singh Chawla, Elsevier probes dodgy citations, Nature 573 (2019) 174.

[48] R. Van Noorden, Journal bans highly cited researcher for citation abuse, Nature 578 (2020) 200201.

[49] S. McLeod, Should authors cite sources suggested by peer reviewers? Six antidotes for handling potentially coercive reviewer citation suggestions, Learn. Publ. doi: 10.1002/leap.1335. 


\section{Supplementary Material}

for

Improved fixed bed models for correlating asymmetric adsorption breakthrough curves

Ronbanchob Apiratikul a , Khim Hoong Chu b,*

${ }^{a}$ Department of Environmental Science, Suan Sunandha Rajabhat University, Bangkok, Thailand

${ }^{\mathrm{b}}$ Honeychem Research, Auckland 1142, New Zealand

${ }^{*}$ Corresponding author.

E-mail addresses: Ronbanchob.Ap@ ssru.ac.th (R. Apiratikul); khimchu@ gmail.com (K.H. Chu)

Overview:

Case S1: Adsorption of lead by waterworks sludge (Table S1, Fig. S1)

Case S2: Adsorption of mercury by nano-adsorbent (Table S2, Fig. S2)

Case S3: Adsorption of nickel by seaweed biosorbent (Table S3, Fig. S3)

Case S4: Adsorption of arsenic by nano-adsorbent (Table S4, Fig. S4)

References

Number of figures: 4

Number of tables: 4 
Case S1: Adsorption of lead by waterworks sludge reported by Faisal et al. [S1]

Table S1. Parameter estimates and goodness of fit statistics for the Bohart-Adams and log-BohartAdams data fits shown in Fig. S1. Model input parameters: $C_{\mathrm{o}}=0.01 \mathrm{mg} \mathrm{cm}^{-3}, L=10 \mathrm{~cm}$, and $u=0.41$ $\mathrm{cm} \min ^{-1}$.

\begin{tabular}{lllll}
\hline Model & $\begin{array}{l}N_{\mathrm{o}} \\
\left.(\mathrm{mg} \mathrm{cm})^{-3}\right)\end{array}$ & $\begin{array}{l}k_{\mathrm{BA}} \\
\left(\mathrm{cm}^{3} \mathrm{mg}^{-1} \mathrm{~min}^{-1}\right)\end{array}$ & $\begin{array}{l}R^{2} \\
(\%)\end{array}$ & RRMSE \\
\hline Bohart-Adams & 2.72 & 0.03 & 92.5 & 0.086 \\
Log-Bohart-Adams & 2.43 & 1.99 & 98.7 & 0.036 \\
\hline
\end{tabular}

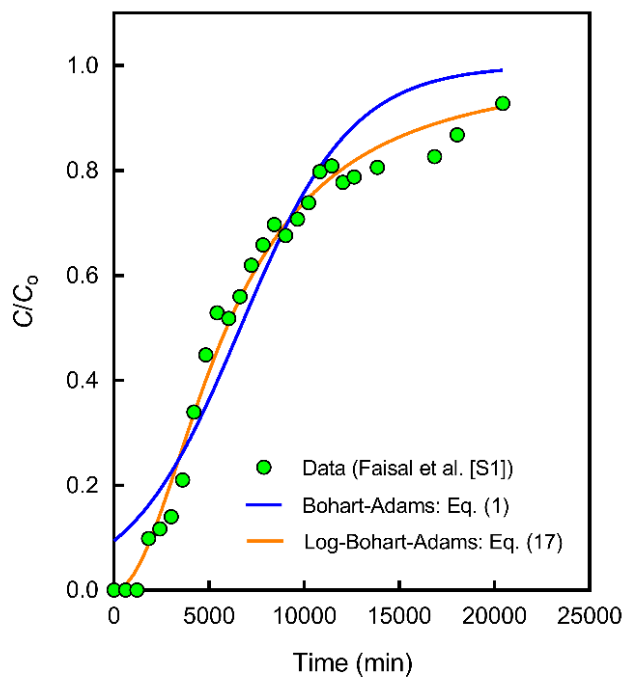

Fig. S1. Bohart-Adams and log-Bohart-Adams data fits compared to observed lead breakthrough data. 
Case S2: Adsorption of mercury by nano-adsorbent reported by Ghasemi et al. [S2]

Table S2. Parameter estimates and goodness of fit statistics for the Bohart-Adams and log-BohartAdams data fits shown in Fig. S2. Model input parameters: $C_{\mathrm{o}}=0.03 \mathrm{mg} \mathrm{cm}^{-3}, L=2.1 \mathrm{~cm}$, and $u=10.2$ $\mathrm{cm} \min ^{-1}$.

\begin{tabular}{lllll}
\hline Model & $\begin{array}{l}N_{\mathrm{o}} \\
\left.(\mathrm{mg} \mathrm{cm})^{-3}\right)\end{array}$ & $\begin{array}{l}k_{\mathrm{BA}} \\
\left(\mathrm{cm}^{3} \mathrm{mg}^{-1} \mathrm{~min}^{-1}\right)\end{array}$ & $\begin{array}{l}R^{2} \\
(\%)\end{array}$ & RRMSE \\
\hline Bohart-Adams & 22.5 & 0.68 & 97.1 & 0.069 \\
Log-Bohart-Adams & 20.5 & 2.93 & 98.7 & 0.046 \\
\hline
\end{tabular}

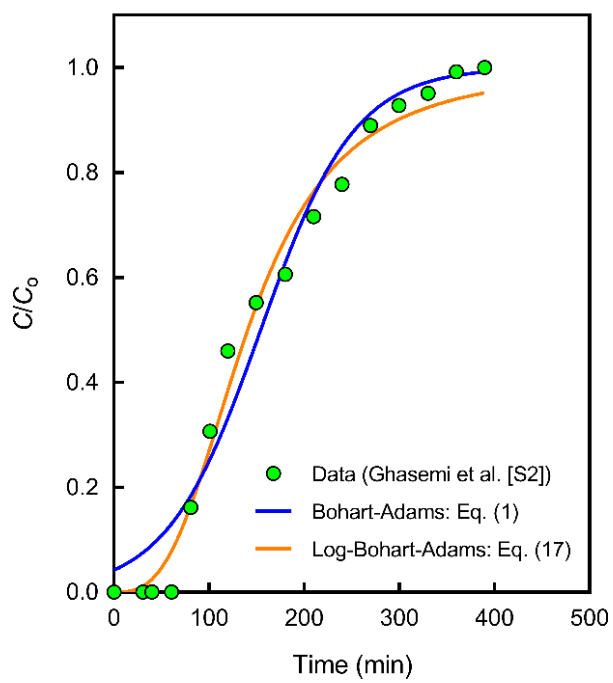

Fig. S2. Bohart-Adams and log-Bohart-Adams data fits compared to observed mercury breakthrough data. 
Case S3: Adsorption of nickel by seaweed biosorbent reported by Barquilha et al. [S3]

Table S3. Parameter estimates and goodness of fit statistics for the Bohart-Adams and log-BohartAdams data fits shown in Fig. S3. Model input parameters: $C_{\mathrm{o}}=0.00049 \mathrm{mmol} \mathrm{cm}{ }^{-3}, L=30.6 \mathrm{~cm}$, and $u=0.65 \mathrm{~cm} \mathrm{~min}^{-1}$.

\begin{tabular}{lllll}
\hline Model & $\begin{array}{l}N_{\mathrm{o}} \\
\left.(\mathrm{mmol} \mathrm{cm})^{-3}\right)\end{array}$ & $\begin{array}{l}k_{\mathrm{BA}} \\
\left(\mathrm{cm}^{3} \mathrm{mmol}^{-1} \mathrm{~min}^{-1}\right)\end{array}$ & $\begin{array}{l}R^{2} \\
(\%)\end{array}$ & RRMSE \\
\hline Bohart-Adams & 0.03 & 2.18 & 99.1 & 0.038 \\
Log-Bohart-Adams & 0.02 & 2.66 & 99.8 & 0.019 \\
\hline
\end{tabular}

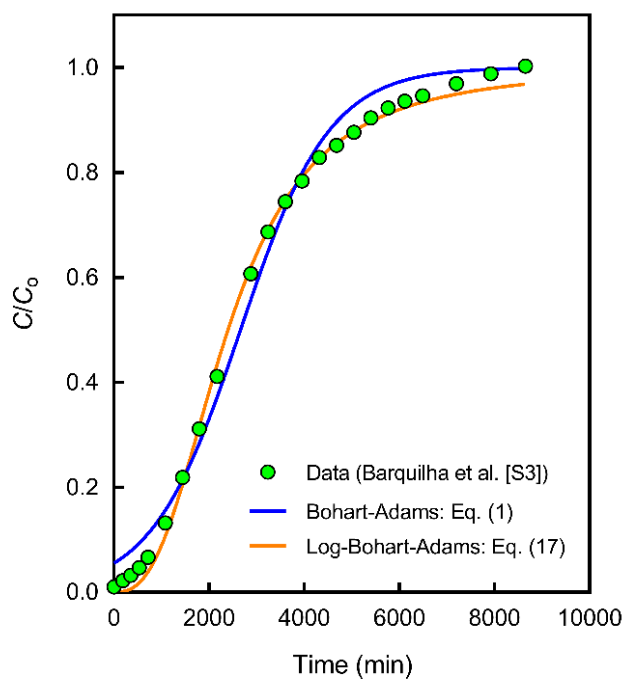

Fig. S3. Bohart-Adams and log-Bohart-Adams data fits compared to observed nickel breakthrough data. 
Case S4: Adsorption of arsenic by nano-adsorbent reported by Contreras et al. [S4]

Table S4. Parameter estimates and goodness of fit statistics for the Bohart-Adams and log-BohartAdams data fits shown in Fig. S4. Model input parameters: $C_{\mathrm{o}}=0.5 \mu \mathrm{g} \mathrm{cm}^{-3}, L=32 \mathrm{~cm}$, and $u=0.35$ $\mathrm{cm} \min ^{-1}$.

\begin{tabular}{lllll}
\hline Model & $\begin{array}{l}N_{\mathrm{o}} \\
\left(\mu \mathrm{g} \mathrm{cm}^{-3}\right)\end{array}$ & $\begin{array}{l}k_{\mathrm{BA}} \\
\left(\mathrm{cm}^{3} \mu \mathrm{g}^{-1} \mathrm{~min}^{-1}\right)\end{array}$ & $\begin{array}{l}R^{2} \\
(\%)\end{array}$ & RRMSE \\
\hline Bohart-Adams & 0.68 & 0.04 & 94.3 & 0.083 \\
Log-Bohart-Adams & 0.61 & 2.23 & 99.2 & 0.031 \\
\hline
\end{tabular}

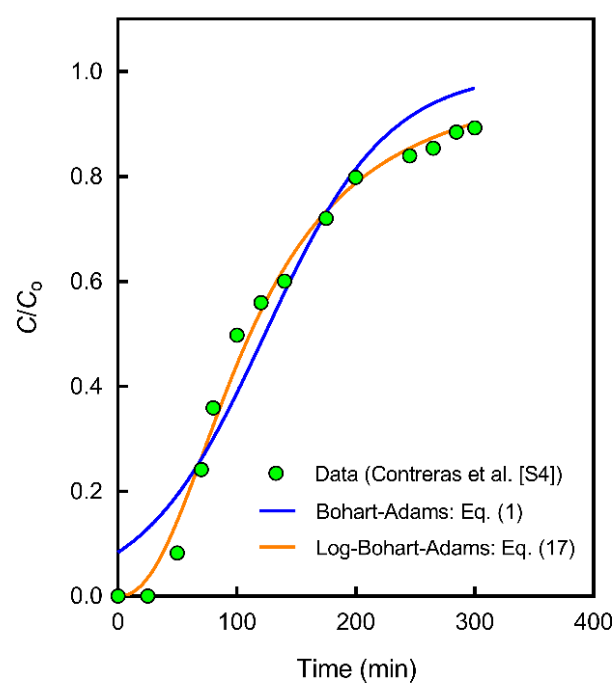

Fig. S4. Bohart-Adams and log-Bohart-Adams data fits compared to observed arsenic breakthrough data. 


\section{References}

[S1] A.A.H. Faisal, S.F.A. Al-Wakel, H.A. Assi, L.A. Naji, Mu. Naushad, Waterworks sludge-filter sand permeable reactive barrier for removal of toxic lead ions from contaminated groundwater, J. Water Process Eng. 33 (2020) 101112.

[S2] S.S. Ghasemi, M. Hadavifar, B. Maleki, E. Mohammadnia, Adsorption of mercury ions from synthetic aqueous solution using polydopamine decorated SWCNTs, J. Water Process Eng. 32 (2019) 100965.

[S3] C.E.R. Barquilha, E.S. Cossich, C.R.G. Tavares, E.A. da Silva, Biosorption of nickel and copper ions from synthetic solution and electroplating effluent using fixed bed column of immobilized brown algae, J. Water Process Eng. 32 (2019) 100904.

[S4] S. Contreras, L. Henríquez-Vargas, P.I. Álvarez, Arsenic transport and adsorption modeling in columns using a copper nanoparticles composite, J. Water Process Eng. 19 (2017) 212-219. 